\title{
Distance decay of similarity, effects of environmental noise and ecological heterogeneity among species in the spatio-temporal dynamics of a dispersal-limited community
}

\author{
Edwige Bellier, Vidar Grøtan, Steinar Engen, Ann Kristin Schartau, Ivar Herfindal and \\ Anders G. Finstad \\ E. Bellier (edwige.bellier@nina.no), A.-K. Schartau and A. G. Finstad, Norwegian Inst. for Nature Research PO Box 5685 Sluppen, \\ NO-7485 Trondheim, Norway. - V. Grotan and I. Herfindal, Centre for Biodiversity Dynamics, Dept of Biology, Norwegian Univ. for Science \\ and Technology, NO-7491 Trondheim, Norway. - S. Engen, Centre for Biodiversity Dynamics, Dept of Mathematical Science, Norwegian Univ. \\ for Science and Technology, NO-7491 Trondheim, Norway.
}

\begin{abstract}
The joint spatial and temporal fluctuations in community structure may be due to dispersal, variation in environmental conditions, ecological heterogeneity among species and demographic stochasticity. These factors are not mutually exclusive, and their relative contribution towards shaping species abundance distributions and in causing species fluctuations have been hard to disentangle. To better understand community dynamics when the exchange of individuals between localities is very low, we studied the dynamics of the freshwater zooplankton communities in 17 lakes located in independent catchment areas, sampled at end of summer from 2002 to 2008 in Norway. We analysed the joint spatial and temporal fluctuations in the community structure by fitting the two-dimensional Poisson lognormal model under a two-stage sampling scheme. We partitioned the variance of the distribution of log abundance for a random species at a random time and location into components of demographic stochasticity, ecological heterogeneity among species, and independent environmental noise components for the different species. Non-neutral mechanisms such as ecological heterogeneity among species $(20 \%)$ and spatiotemporal variation in the environment $(75 \%)$ explained the majority of the variance in log abundances. Overdispersion relative to Poisson sampling and demographic stochasticity had a small contribution to the variance (5\%). Among a set of environmental variables, lake acidity was the environmental variable that was most strongly related to decay of community similarity in space and time.
\end{abstract}

Environmental variability, ecological heterogeneity among species (Lande et al. 2003) as well as demographic stochasticity and dispersal of individuals (Hubbell 2001) are important factors affecting the coexistence of species within a community and $\beta$-diversity in space and time. The joint spatial and temporal fluctuations of relative log abundances induce a decay in community similarity with geographic distance (Nekola and White 1999). Similarity is sensitive to key spatial processes such as dispersal and spatial variation in environmental conditions. Analyses of how the similarity of communities varies with geographic distance can be used to understand spatial patterns in biological diversity and community evolution (Condit et al. 2002, Gilbert and Lechowicz 2004, Soininen et al. 2007, Maloney and Munguia 2011, Astorga et al. 2012). For example, the shape of the landscape affects the dispersal rates of organisms among localities. Community similarity decreases faster in a landscape with strong dispersal barriers than within open areas (Soininen et al. 2007).

Similarity can decay because of differences in environment among localities. Niche theory predicts that relative abundances of species will change as a result of speciesspecific differences in evolved adaptive responses to environmental gradients (Gilbert and Lechowicz 2004). The ecological niche of a species can be viewed as an environmental volume where the axes are abiotic and biotic factors influencing birth and death of the individuals of that species (Hutchinson 1957). This may cause ecological heterogeneity among species due to differences in their growth rates in a given environment. Autocorrelated spatial and temporal variation in environmental conditions will cause autocorrelated spatial and temporal fluctuations in species abundance and presence (Engen et al. 2002) if there is among species variation in adaptations to different environments. Many studies, both theoretical (Ranta et al. 1997a, b, 1999, Lande et al. 1999) and empirical (Ranta et al. 1995, Lindstrom et al. 1996, Grenfell et al. 1998, Bjørnstad et al. 1999, Engen et al. 2005, Grøtan et al. 2005, Sæther et al. 2007) show that fluctuations in abundance and/or vital demographic parameters are spatially synchronised and that the synchrony in abundance decreases with increasing geographical distance between populations. 
The scale of synchrony depends on the scale of spatially correlated environmental fluctuations through the Moran effect (Moran 1953) and individual dispersal distances (Lande et al. 1999). Knowledge of the spatial scale of synchrony is important because it influences the risk of local and global extinction (Allen et al. 1993, Bolker and Grenfell 1993, Heino et al. 1997). Engen and Sæther (2005) generalized the Moran effect and showed how spatial environmental covariation can synchronize fluctuations of spatially segregated populations with no interchange of individuals even if the dynamics are nonlinear. The influence of the Moran effect and dispersal on synchrony of community dynamics has been poorly empirically studied. This is more complex than for single species because the fluctuations in abundance of different species depend on interspecific interactions. Theory and empirical studies suggest that the loss or decline of one species can be compensated by the growth of another (Ives and Cardinale 2004, Vasseur et al. 2005, Vasseur and Gaedke 2007). Compensatory dynamics is difficult to detect because population dynamics are governed by the direct effects of environmental fluctuations on demography and species interactions (Lande et al. 2003, Ranta et al. 2008). Some recent studies suggest that the effects of competition on temporal fluctuations in species abundances is weak compared to effects of environmental variation (Houlahan et al. 2007, Mutshinda et al. 2011).

In contrast to niche theory, neutral theory (Caswell 1976, Bell 2001, Hubbell 2001, Etienne 2007, Etienne et al. 2007, Rosindell et al. 2011) assumes ecological equivalence among species and that demographic stochasticity combined with spatially limited dispersal causes the decay of community similarity. Demographic stochasticity causes random spatial and temporal variation in the species relative abundances independently of the other factors. The effect of demographic stochasticity depends heavily on dispersal rates and dispersal distances as dispersal will counteract local drift in abundances (Hubbell 2001). The scale of decay of similarity will depend of the dispersal rates of the individuals of the communities. In the extreme case of no dispersal among local communities, demographic stochasticity will be the only factor causing temporal turnover in species relative abundances and there will be no spatial autocorrelation in community similarity. The contribution of demographic stochasticity to fluctuations in abundance is inverse density-dependent, and in large communities (Lande et al. 2003) the rate of temporal turnover could be extremely slow.

Zooplankton species inhabiting lentic freshwater habitats offer an excellent model system to analyse decay of similarity and dynamics of fragmented community in a natural system. The island-like nature of lakes creates welldefined habitat boundaries for the community (Jenkins and Underwood 1998, Shurin 2000, Bohonak and Jenkins 2003, Leibold et al. 2004). Zooplankton communities in lakes located in independent watershed are only connected by overland dispersal that is not frequent on relatively short time scales (Bohonak and Jenkins 2003). Species composition of very small organisms communities in nonconnected lake might partly result of age isolation (Vyverman et al. 2007). Thus, zooplankton abundance data from communities where dispersal between localities is absent simplifies interpretation of the distance decay of similarity. Such data provides unique and crucial insights with respect to what extent spatially autocorrelated environmental fluctuations contribute to spatial fluctuations in relative abundances of species. In this study, we use zooplankton community data from 17 lakes in Norway (Fig. 1) located in hydrologicaly isolated catchment areas and covered by ice almost half of the year. The data were collected between 2002 and 2008 at the end of the summer. This zooplankton community is composed of species with a rather long generation time mainly due to ice-period (Halvorsen et al. 2004), generally 1 year for copepods and 2-4 months for cladocerans. Zooplankton communities represent a critical link between primary
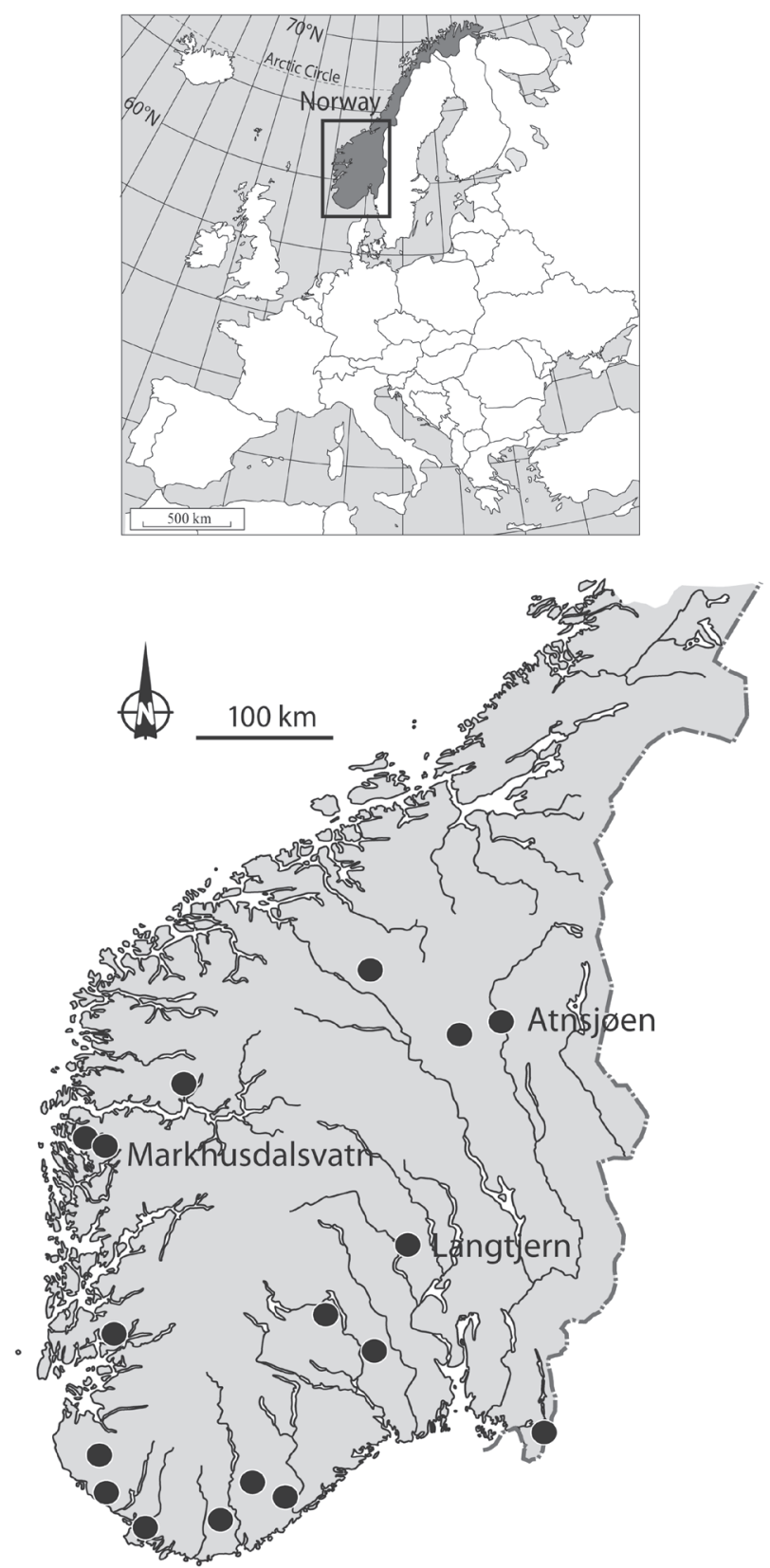

Figure 1. Localisation of the 17 lakes in Norway included in this study. 
producers and high consumers in aquatic food webs and responds to environmental variability (Shurin et al. 2010). Time series plots of the most common species in 3 of the lakes (Fig. 2) show both spatial (among lakes) and temporal (within lake) fluctuations in species abundances. These fluctuations may be due to spatiotemporal variation in environmental conditions, demographic stochasticity and ecological heterogeneity among species. In this article, we use statistical techniques that estimate the relative contributions of these factors with respect to spatiotemporal variation in relative log abundances (Engen et al. 2002) in order to better understand the processes that lead to differences among species in relative abundance when migration of individuals among communities is low. If

(a)

Markhusdalsvatn

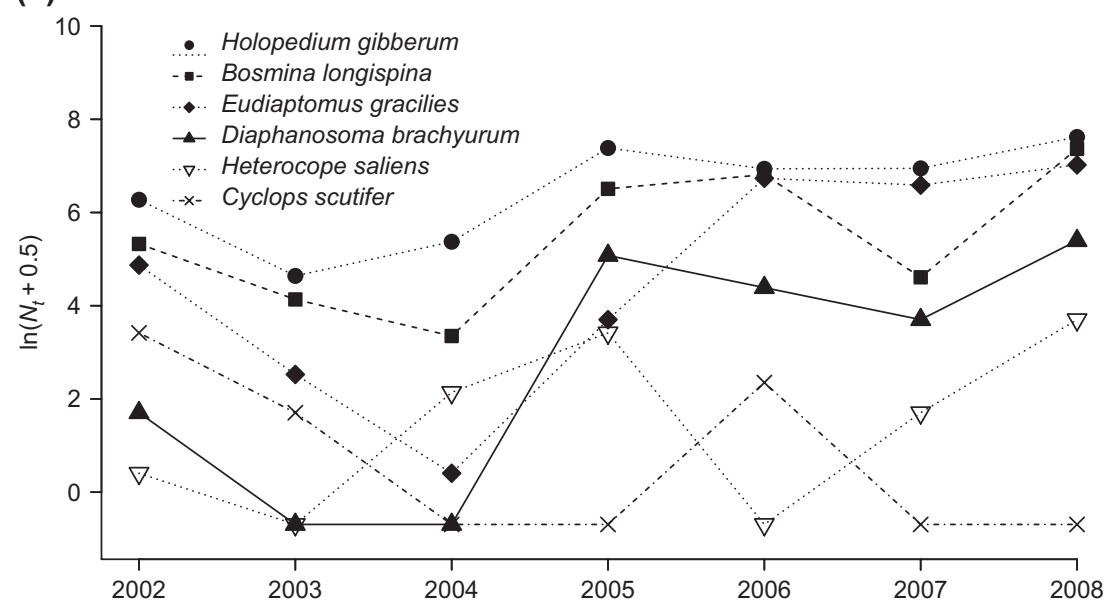

(b)
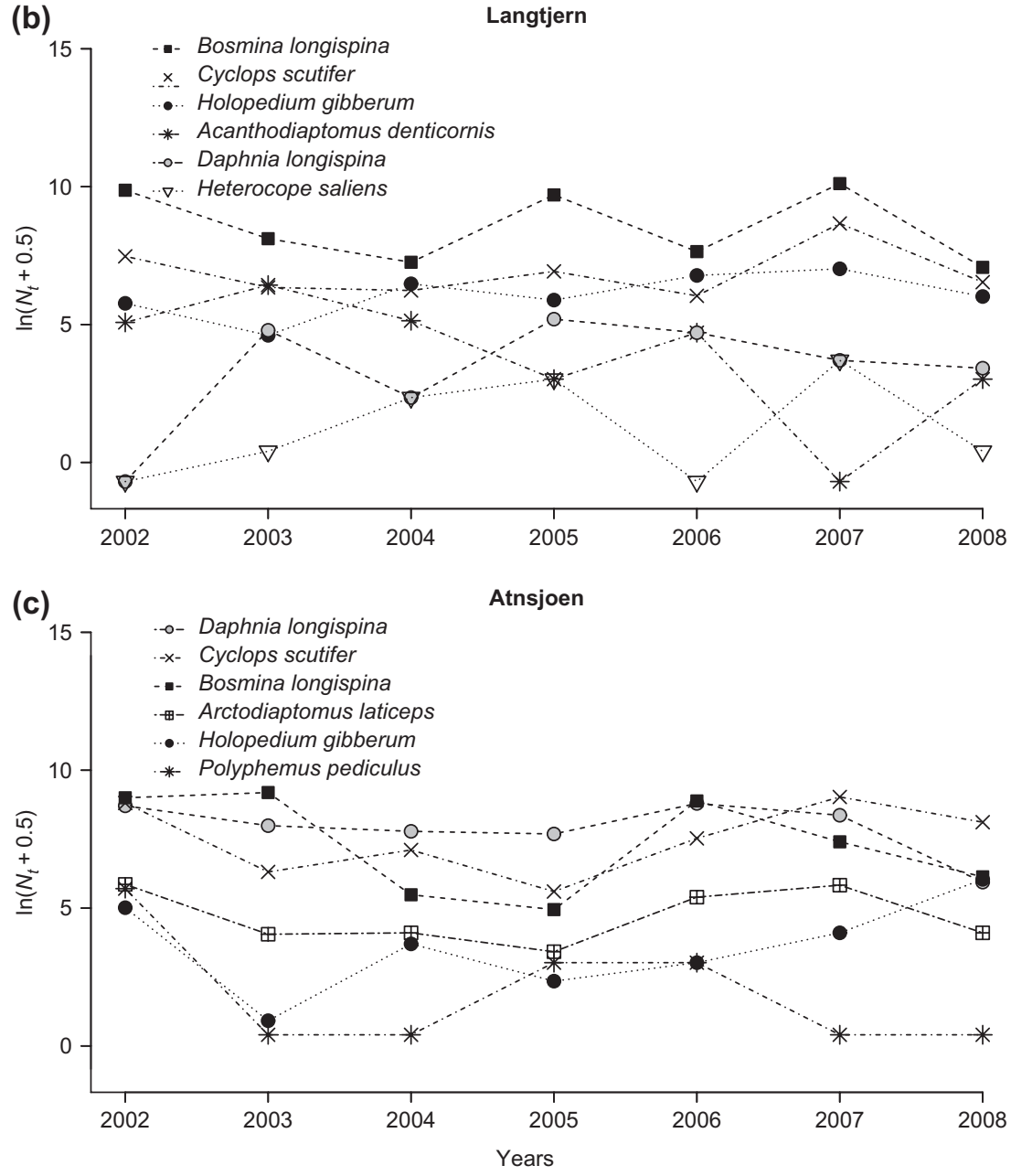

Figure 2. Times series of $\log$ abundance $\ln \left(N_{t}+0.5\right)$ for the 6 most abundant species, where $N_{t}$ is the observed count at time $t$ of the lakes Markhusdalsvatn (a), Langtjern (b), Atnsjøen (c). The locations of these 3 lakes are shown with names in Fig. 1. 
community dynamics is influenced by species-specific niches, we will expect to find large contributions from ecological heterogeneity among species and spatio-temporal variation in environmental conditions. The contribution due to the heterogeneity in growth rates and carrying capacities among species reflects innate differences in the biology of each species that influence species abundance and contradicts the assumption of ecological equivalence of species made in neutral models. Our study sheds light on the relative importance of the factors of the community dynamics of highly fragmented communities and gives insight on how fragmentation of habitats may affect species coexistence in fluctuating environments.

\section{Material and methods}

\section{The model}

The joint spatial and temporal fluctuations in the community structure are analysed by fitting the bivariate Poisson lognormal distribution (Engen et al. 2002) to a large number of observations in space and time. By applying multivariate dependent diffusion for describing the fluctuations in abundances (Engen and Lande 1996), the variance in the lognormal species abundance distribution can be partitioned into components expressing the dynamics of the community (Engen et al. 2002, Lande et al. 2003).

\section{Distance decay of similarity}

When considering two communities jointly, either at two different locations or within the same community at two different time points, we assume that the log abundances in the pair of communities have the binormal distribution so that the lognormal model still fits each community separately. The correlation of this distribution then serves as a measurement of similarity between communities (Engen et al. 2011b, Sæther et al. 2013). In this study, we estimate similarity under a two-stage sampling scheme and extend the approach that combines counts and incidence data to estimate the univariate Poisson lognormal species abundance distribution (Bellier et al. 2012) to the bivariate lognormal species abundance distribution. We describe in Supplementary material Appendix A how we find the maximum likelihood estimators for the parameters of the bivariate Poisson lognormal distribution based on samples that are collected and processed as described in the following. First, a species that has log abundance $x$ in the community will be represented in a sample with sampling intensity $v$ by a number of individuals that is Poisson distributed with mean $v e^{x}$. However, this sample is considered too large to make counting of all individuals possible ('Data collection and environmental variables'). Therefore, a small sample that constitutes a fraction $q<1$ of the sample is drawn at random. All individuals are counted in the $q$ fraction of the sample. Some species with a small abundance are likely to be missing in the small sample but to be present in the larger sample. Such rare species provide important information about the left tail of the marginal distributions as well as the correlation among communities. We modified the function bipoilogMLE of the R-package (R Development
Core Team) poilog (Grøtan and Engen 2008) to estimate the bivariate Poisson lognormal species abundance distribution by combining such count and incidence data. To reveal distance-decay of similarity (Nekola and White 1999), all pair-wise estimates of the correlation parameter of the twodimensional Poisson lognormal species abundance distribution can be plotted against distance.

\section{Partitioning of the variance of the lognormal species abundance distribution}

To partition the total variance of $\log$ abundance, the bivariate lognormal model (Engen et al. 2002, 2011b) is linked to a stochastic dynamic model that generates the lognormal species abundance distribution (Engen and Lande 1996). The model is based on a general stochastic theory, with colonization, speciation, extinction, density regulation and environmental stochasticity (Engen and Lande 1996, Engen et al. 2002, 2011a,b). The model allows species abundance to change due to species-specific as well as common environmental stochasticity in the density-independent growth rate $r$ and due to deterministic density dependence acting within species (Supplementary material Appendix B). These factors cause temporal and spatial fluctuations in relative abundances. Engen and Lande (1996) showed that different forms of density dependence within species produces different shapes of species abundance distributions. Assuming a Gompertz density dependence and constant environmental variances acting independently on each species produce a lognormal species abundance distribution. Engen et al. (2002) included ecological heterogeneity among species modeled by among-species variation in $r$, showing that the variance of the lognormal species abundance distribution can be decomposed into three additive components (Supplementary material Appendix B, Eq. 4). First, there is an environmental component due to independent environmental noise terms with variance $\sigma_{s}^{2}$. The second term is the demographic component with variance $\sigma_{d}^{2}$, and the last term is the interspecies ecological heterogeneity component due to the ecological heterogeneity among the species determined by the variance $\sigma_{r}^{2}$ of $r$ among species. Under the assumption of neutral community dynamics without dispersal, only demographic stochasticity should contribute to the total variance of the species abundance distribution as there is no environmental noise. If the sampling is overdispersed compared to the Poisson distribution, this will increase the estimated variance. The link between the statistical model and the stochastic model is established through the spatial correlation in the noise generating the correlation in the bivariate lognormal distribution. We assume that the environmental noise has a spatial structure, is acting independently on each species and is expressing independent stochastic fluctuations in time and space in reproductive rates and mortality of the species in the community. From the spatio-temporal model (Supplementary material Appendix B, Eq. 6), it is possible to estimate the strength of intraspecific dependence, the scale of spatial autocorrelation in environmental stochasticity and the three components of the variance of the species abundance distribution. The variance of $\log$ species abundance can be assumed to be the same at all sampling 
sites and times (Engen et al. 2002, Lande et al. 2003) and was estimated by averaging the estimated variances obtained by fitting univariate Poisson lognormal distributions to the observed species abundance distribution in each sample (Lande et al. 2003). The three components of the variance of $\log$ species abundance within sites and times can be estimated by decomposing (Supplementary material Appendix B, Eq. 6) into additive components, $A=\frac{\sigma_{s}^{2} \rho_{\infty}}{\sigma_{s}^{2}+\sigma_{d}^{2} / K+2 \sigma_{r}^{2} / \delta}$ $B=\frac{\sigma_{s}^{2}\left(1-\rho_{\infty}\right)}{\sigma_{s}^{2}+\sigma_{d}^{2} / K+2 \sigma_{r}^{2} / \delta} \quad$ and $\quad C=\frac{2 \sigma_{r}^{2} / \delta}{\sigma_{s}^{2}+\sigma_{d}^{2} / K+2 \sigma_{r}^{2} / \delta}$ The sum of $A$ and $B$ gives an estimate of the environmental component of the variance of the lognormal species abundance distribution, $\mathrm{C}$ is the component due to ecological heterogeneity among species and the demographic component is equal to $1-(A-B)$. The variance of each correlation estimate is large and varies in an unknown way with sample sizes and the magnitude of the true correlations (Engen et al. 2002, 2011b, Grøtan et al. 2012). Because this information is not available, we chose to fit the spatio-temporal model (Supplementary material Appendix B, Eq. 6) by simple least squares. All confidence intervals are based on 1000 resamples with replacement of the residuals of the model (Efron and Tibshirani 1993).

\section{Partitioning of the environmental noise}

To identify the environmental variables which are essential in shaping the community structure in space and time, we split the environmental stochasticity into components expressed by environmental covariates (Supplementary material Appendix B, Eq. 7). Hence, autocorrelation is a decreasing function of a generalized distance measure expressing differences between communities in term of standardized environmental dissimilarity $W$. The generalized distance is expressed as $v+\beta W$ where $v$ is the distance between locations of pairs of communities. The contribution to the distance-decay can be estimated by assuming that the expectation of $E(v+\hat{\beta} W)$ is linear in $v$. This allows us to estimate the linear generalized distance model of the form $E(v+\hat{\beta} W)=a+\Lambda v$ where $a$ is the intercept and $\Lambda$ is the slope. If the slope $\hat{\Lambda}$ is equal to 1 , the expectation of $W$ does not depend on $v$. Thereby, the mean effect of an environmental dissimilarity is equal to the intercept $\alpha$ of the generalized distance model. The mean effect of an environmental dissimilarity can also be estimated as $H=\hat{\beta} E(W)$ by multiplying the expectation of the dissimilarity by the estimated $\hat{\beta}$. The estimate of $H$ gives an estimation of the contribution to the distance decay in similarity caused by spatial variation in a given environmental variable.

\section{Data collection and environmental variables}

The assessment of the spatial and temporal fluctuations of freshwater zooplankton communities was based on a monitoring data set collected in Norway (Walseng et al. 2006). The data were collected in the pelagic area of 17 independent lakes from 2002 to 2008 in late summer (mid-August to mid-September). Samples were processed following a two-stage sampling procedure. First, a smaller sample was drawn from the sample and all individuals were identified to species and counted (Downing and Rigler 1984). Thereafter, the total sample was screened to identify the species not represented in the small sample. All crustaceans were identified to species; rotifers were not included in this survey. We selected a set of relevant covariables according to the results of a principal component analyses performed on a set of weather and chemical data (the mean and variance of the temperature in July and August, precipitation in July and August, the snow depth in April, May and June, altitude, the $\mathrm{pH}$ and total organic carbon) that can affect freshwater zooplankton diversity (Hessen et al. 2006, Shurin et al. 2010). For each sampling occasion, the $\mathrm{pH}$ and the total organic carbon (TOC) were measured in each lake according to standard NS 4720 and NS-EN 1484, respectively. The $\mathrm{pH}$ and organic carbon are linked variables, but have different effects on the zooplankton community. The $\mathrm{pH}$ influences the diversity of the zooplankton community whereas the concentration of organic carbon increases the abundance of daphnids over other cladocerans (Yan et al. 2007) and affects the evenness of the community. Weather data were obtained from the Norwegian Meteorological Inst. (<http://met.no/ $>$ ). The data included downscaled and gridded daily maps of temperature, precipitation and snow depth with a $1 \mathrm{~km}^{2}$ horizontal resolution in the period 2002-2008 (Skaugen et al. 2003). We first extracted weather data based on coordinates of the lakes. Thereafter, the daily data were averaged or summed to monthly data. The temperature in July and August are linked as well but have a different time lag effect on the community diversity (Thompson and Shurin 2011). The snow depth is an indicator of when the spring arrives, and precipitation affects the flow of the streams feeding the lakes.

\section{Results}

\section{Distance-decay of similarity}

The similarities for different time lags were plotted against distance to reveal the pattern of distance-decay, and similarity decreased substantially with increasing distance among lakes (Fig. 3). The decay was well-evident from a distance of zero to a distance of $\sim 150 \mathrm{~km}$. The spatial scale of intraspecific spatio-temporal autocorrelation was estimated as $\hat{l} \sim 60 \mathrm{~km}$ for time lag 0 (Supplementary material Appendix B, Eq. 6, Table 1). This means that the environmental noise acting on a single species at locations separated by a distance of less than $60 \mathrm{~km}$ will have a correlation larger than $e^{0.5} \approx 0.6$, while the correlation will practically vanish at a distance of about $180 \mathrm{~km}$ (Table 1, Fig. 4). The spatial scale of population autocorrelation decreased with increasing time lag between samples (Fig. 4).

\section{Variance partitionning of the log-abundance}

The estimated variance of relative $\log$ abundances (Supplementary material Appendix B, Eq. 4), when pooling all data into a single sample was $\hat{\sigma}^{2}=11.83$ with estimated components shown in Fig. 5a. The estimate of the 


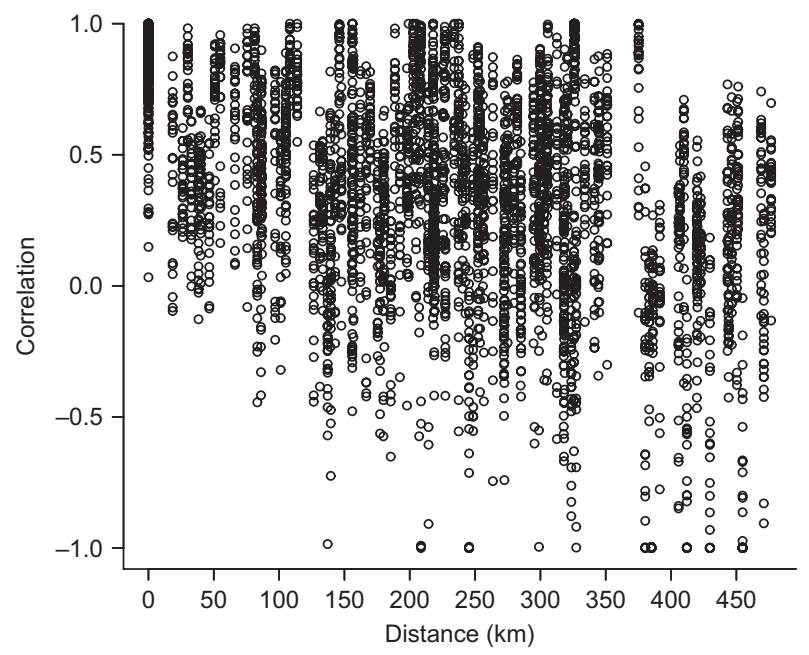

Figure 3. Estimated correlations for all time differences from 0 to $5 \mathrm{yr}$ plotted against spatial distance in kilometers.

component due to environmental variation acting independently on each species of the community was $\hat{\sigma}_{e}^{2}=8.87$ and explained $75 \%$ of the total variance (Fig. $5 \mathrm{~b}$ ). The estimate of variance due to ecological heterogeneity among species $\left(\hat{\sigma}_{r}^{2}=2.37\right)$ indicated a substantial amount of variation in growth rates leading to among species variation in carrying capacities. Although the interspecies ecological heterogeneity component was large in absolute terms, the relative contribution of this component was moderate (20\%) due to the large environmental variance component. Finally, demographic stochasticity $\left(\hat{\sigma}_{d}^{2}=0.59\right)$ explained only $5 \%$ of the variance of $\log$ abundances. This estimate may also include an effect of overdispersion in the sampling distribution relative to the Poisson distribution. The local strength of density regulation was $\hat{\delta}=0.07(0.04,0.089)$. The return time to equilibrium is $1 / \hat{\delta}$, meaning that the species in the community are expected to pass their equilibrium abundance each 14 th year on average.

\section{Composition of the environmental noise}

Based on the estimates of the parameters of Eq. 4 (Supplementary material Appendix B), we can predict the change in similarity due to a change in standardized environmental variables. The change in similarity $R$ at time lag 0 and distance 0 predicts the expected difference in similarity between a pair of communities that experience the same environmental conditions and a pair of communities that experience different environmental conditions due to a single environmental variable that differs among the two communities. The dissimilarity in $\mathrm{pH}$ had the largest effect $(\hat{R}=0.44)$ on $p(0,0)$ while others environmental variables had almost the same effect $(\hat{R} \sim 0.35$, Table 1$)$. Estimate of $H$ (Fig. 6, Table 2) gives an estimation of the contribution of the spatial variation of an environmental variable to the distance decay of community similarity. Dissimilarities of $\mathrm{pH}$ had the largest effect on decay of community similarity. August and July temperature and the summer precipitation had smaller effects. The snow depth and the total organic carbon had almost no effects on the similarity decay.

\section{Discussion}

\section{Distance-decay with dispersal limitation}

Environmental autocorrelation (Moran effect) decreases with distance which consequently causes an associated decrease in community similarity. Lande et al. (1999) who analyzed in theoretical models how different factors are expected to affect the spatial scaling of the population sizes, showed that relative to environmental correlation, the contribution of individual dispersal to the spatial scale of synchrony is increased by the ratio of the individual dispersal to the strength of density regulation. If the scale of individual dispersal is smaller than that of environmental correlation, dispersal can substantially increase the scale of population synchrony for weakly regulated population. In this study, dispersal can not act to increase the spatial scale of community similarity decay because the zooplankton communities are not connected and dispersal of individuals may be only very low. However, the zooplankton community is weakly density regulated $\hat{\delta}=0.07(0.04,0.089)$ and dispersal of individuals could have an effect on the scale of similarity decay.

\section{Components of the community dynamics}

The species specific environmental noise explains a large fraction $(75 \%)$ of the total variance in the species

Table 1. Estimated values of the parameters of the autocorrelation function (Eq. 4) with only the distance and with different environmental dissimilarities $W$ and the observed mean of $W$. The $95 \%$ intervals obtained from bootstraping the residuals are given in parentheses. Estimates of $\hat{R}$ are equal to $\rho(0,0, W=0)-\rho(0,0, W=1)=1-B \mathrm{e}^{-1 / 2 * \hat{\beta} / \|^{2}}$ and correspond to a reduction in $\rho(0,0)$ due to one standard deviation change of the standardized covariate. The estimates of spatial scale / are in $\mathrm{km}$.

\begin{tabular}{|c|c|c|c|c|c|c|c|}
\hline & $\hat{A}$ & $\hat{B}$ & $\hat{\imath}$ & $\hat{\delta}$ & $\hat{\beta}$ & $\begin{array}{c}\text { Mean of } \\
(\hat{W})\end{array}$ & $\hat{R}$ \\
\hline list? & $0.09(0.09-0.96)$ & $0.65(0.66-0.63)$ & $59(55-64)$ & $0.07(0.04-0.093)$ & - & - & - \\
\hline Tem! & $11(0-0.16)$ & $66(0.64$ & $57-$ & $06(0.03-$ & $13.81(12.05$ & 1.03 & \\
\hline Temperature in August & $0.11(0-0.16)$ & $66(0.64$ & $69(57-$ & $06(0.012-$ & $13.81(12.05$ & 1. & 0.3 \\
\hline Precipi & 0.1 & & $67(65-$ & ) & 15. & 1.06 & \\
\hline Snow depth in April & $0.10(0-0.29)$ & $.66(0.64-0.74)$ & $62(57-68)$ & $0.06(0.03-$ & $9.05(8.07-13$ & 1.01 & 0.3 \\
\hline $\mathrm{pH}$ & $0.12(0-0.27)$ & $0.70(0.64-0.74)$ & $126(116-138)$ & $0.05(0.03-0.069)$ & $82.34(80.07-85.98)$ & 1.01 & \\
\hline Total organic carbon & $0.09(0-0.32)$ & $0.66(0.64-0.74)$ & $72(56-75)$ & $0.07(0.04-0.09)$ & $14.35(12.07-16.98)$ & 1.00 & 0.3 \\
\hline
\end{tabular}




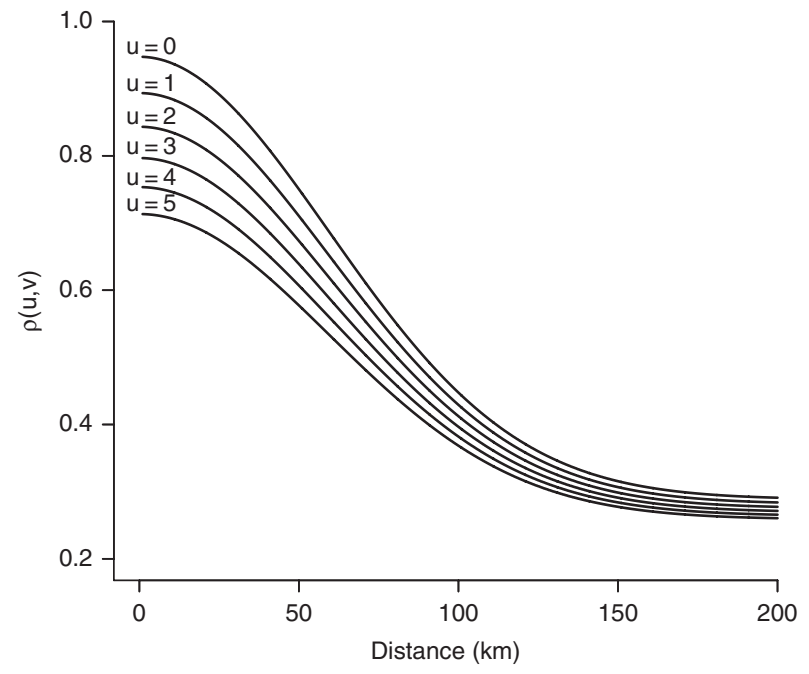

Figure 4. Curves of the fitted spatio-temporal covariance model (given by Supplementary material Appendix B, Eq. 3) from 0 to 5 yr time lag.

abundance distribution for the freshwater zooplankton community (Fig. 5). Previously, the parameters in the model of Engen and Lande (1996) have been estimated for a community of tropical butterflies (Engen et al. 2002, Lande et al. 2003). Zooplankton and butterfly community are both composed of many species with interspecific variation in life history. Zooplankton communities in sub-Arctic lakes are composed of species with life cycles ranging from $1 \mathrm{yr}$ to 2 months and the mean generation time of species in the butterfly community is around $2-5$ months. Here, we compared the estimated parameters of the interannual spatio-temporal dynamics of zooplankton and tropical butterflies because both communities are composed of species with similar mean generation times. Our environmental component is much larger $\left(\hat{\sigma}_{e}^{2}=8.87\right)$ than the environmental component (Fig. 5) estimated for the butterfly community $\left(\hat{\sigma}_{e}^{2}=0.48\right)$ by Engen et al. (2002). The estimate of the variance component due to ecological heterogeneity among species, representing variation in specific growth rate and local carrying capacity, was similar to the estimate obtained in the butterfly community $\left(\hat{\sigma}_{r}^{2}=2.37\right.$ versus $\left.\hat{\sigma}_{r}^{2}=2.75\right)$. This result is in disagreement with the assumption of ecological equivalence of species of the neutral model. If $\hat{\sigma}_{r}^{2}=0$ this community is neutral in the sense that each species has the same dynamics driven by environmental noise only. The relative contributions of the interspecific heterogeneity to the total variance of log abundances are however different, $19 \%$ for the zooplankton community versus $80 \%$ for the tropical butterfly community. The variance in abundance among species of zooplankton is mainly due to environmental stochasticity generated by inter-annual fluctuations in vital rates while for the tropical butterfly the variance was mainly due to ecological heterogeneity among species. These differences may be due to differences in density-regulation, the zooplankton community was weakly density-regulated while the density-regulation of the butterfly community was very large $\left(\hat{\delta}=1.61 \mathrm{yr}^{-1}(0.58,2.63)\right.$ (Engen et al. (a)
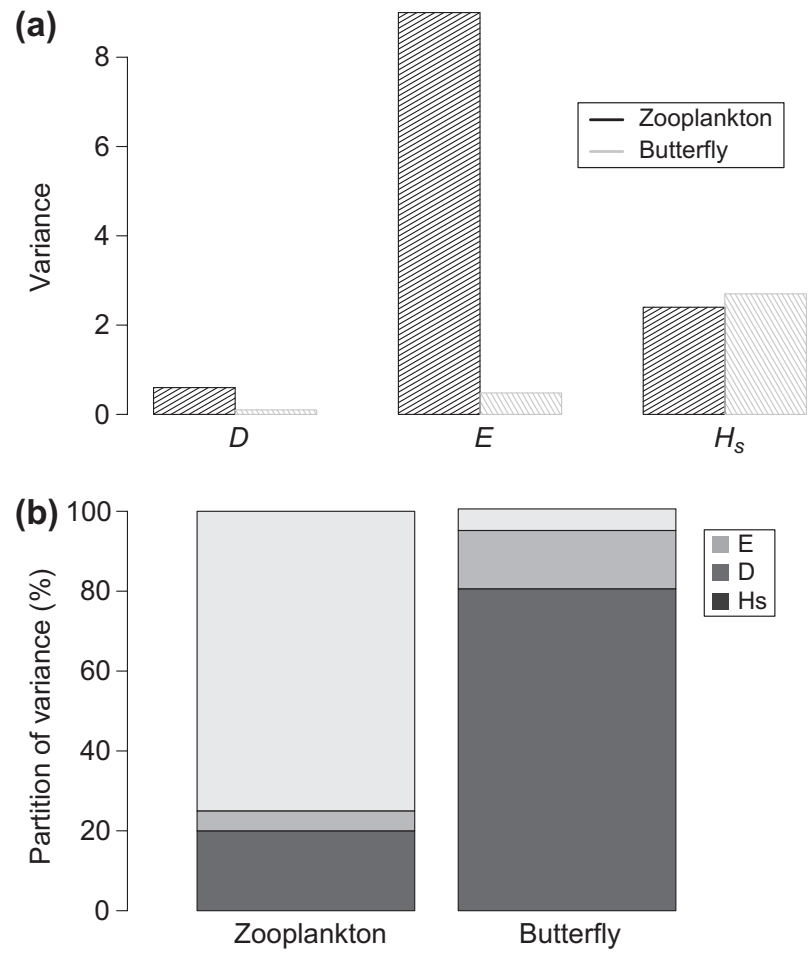

$H_{s}$

Figure 5. (a) Estimated components of variance in log abundance in a freshwater zooplankton community and a tropical butterfly community (Table 8.1, (Lande et al. 2003)). (b) Estimated partitioning of the variance of the lognormal species abundance distribution into three components in our zooplankton community (left), the environmental component $(E)$ explained $75 \%$, the ecological heterogeneity among species $\left(H_{s}\right)$ explained $20 \%$ and the demographic component $(D)$ explained $5 \%$. In a tropical butterfly community. (right), the environmental component $(E)$ explained $5.4 \%$, the ecological heterogeneity among species $\left(H_{s}\right)$ explained $80.6 \%$ and the demographic component $(D)$ explained $14 \%$ (Engen et al. 2002).

2002)). For the zooplankton community, the general environmental stochasticity tends to occur as medium scales events that are not rapidly damped (Fig. 4) due to weak density regulation. The large value of the environmental component is associated with a weak density-regulation suggesting that environmental variability produces temporal fluctuations in species diversity. When communities are isolated by strong dispersal barriers, exchange of individuals cannot act to homogenize the composition and structure of the communities. Individuals that are maladapted to new environmental conditions cannot be replaced by immigrant individuals with favoured traits. Communities disturbed by habitat fragmentation may be less resistant in the face of climate change or other anthropic disturbances (Travis 2003).

\section{Composition of the environmental noise}

The effects of the covariates (measured by $R$ and $H$, Table 1 , Fig. 6) showed that at a regional scale the main variables that cause similarity in space and time are the $\mathrm{pH}$, temperature and precipitation in July and August. At 

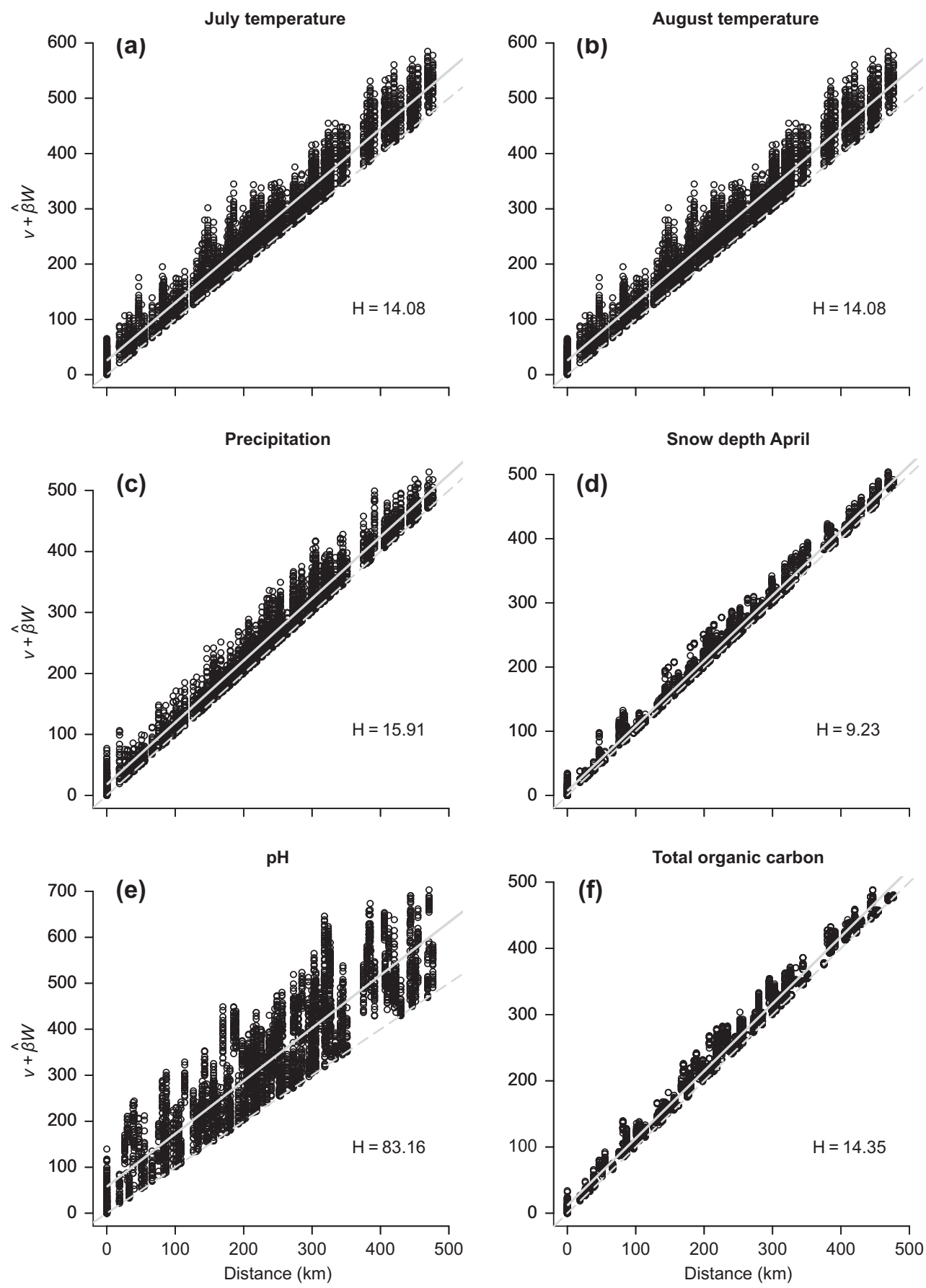

Figure 6. Regression line (solid line) of the generalized linear distance model $E(v+(\beta \hat{W})=\alpha+\Lambda v$ fitted for different standardized environmental dissimilarities $W$. The dotted line corresponds to the null model when $\beta=1$. The points are the estimated generalized distance values $v+(\beta \hat{W})$. The contribution to distance decay in similarity caused by spatial variation in a given environmental variable is estimated such as $H=\hat{\beta} E(W)$.

local scale, $\mathrm{pH}$ was the most important variable in shaping the community structure in space and time. Acidification is known to have a major effect on the structure and dynamics of aquatic systems and particularly zooplankton communities (Klug et al. 2000, Fischer et al. 2001, Cottenie 2005, Steiner et al. 2011) and can vary greatly both spatially and temporally due to natural and anthropogenic processes (Keitt 2008, Steiner et al. 2011). From an experimental study, Steiner et al. (2011) showed that acidification events decreased zooplankton species diversity by decreasing both species richness (via extinctions) and evenness. When the evenness of a community decreases, some species become more common (i.e. more dominant) while others become more rare. Dominance may affect the coexistence of regional species by altering community dynamics and compositional stability 
Table 2. Estimation of the intercept $\hat{a}$ and the slope $\hat{\Lambda}$ of the generalized distance model. We tested if the intercept $\hat{a}$ was different from 0 and if the slope $\hat{\Lambda}$ was different from 1 with a student test.

\begin{tabular}{|c|c|c|c|c|c|c|c|}
\hline \multirow[b]{2}{*}{ Environmental variables } & \multirow[b]{2}{*}{ DF } & \multicolumn{3}{|c|}{ Intercept } & \multicolumn{3}{|c|}{ Slope } \\
\hline & & $\hat{a}$ & t-statistics & $\mathrm{p}$ & $\hat{\Lambda}$ & t-statistics & $\mathrm{p}$ \\
\hline Temperature in July & 4156 & 25.05 & 31.67 & $<0.001$ & 1.04 & 18.18 & $<0.001$ \\
\hline Temperature in August & 4156 & 25.06 & 31.67 & $<0.001$ & 1.04 & 12.90 & $<0.001$ \\
\hline Precipitation in August & 4156 & 17.19 & 30.99 & $<0.001$ & 1.01 & 4.54 & $<0.001$ \\
\hline Snow depth in April & 4156 & 6.83 & 20.07 & $<0.001$ & 1.01 & 7.55 & $<0.001$ \\
\hline $\mathrm{pH}$ & 4156 & 57.56 & 29.98 & $<0.001$ & 1.14 & 18.76 & $<0.001$ \\
\hline Total organic carbon & 4156 & 11.57 & 29.01 & $<0.001$ & 1.01 & 6.26 & $<0.001$ \\
\hline
\end{tabular}

(Hillebrand et al. 2008). Our results support also the hypothesis that zooplankton communities are very sensitive to variability in temperature in late summer. Indeed, warming temperatures in August can increase productivity (Thompson and Shurin 2011) and other studies have documented differential zooplankton responses to temperature (Achenbach and Lampert 1997, Beninca et al. 2011). Temperature can also affect generation time of zooplankton that is important determinant of the rate of population growth (Gillooly 2000). Variation in temperature may have effects on the structure of the zooplankton community. This could have complex consequences on the functioning of ecological systems of lakes (Keller 2007). In our study, the dynamics of our zooplankton communities are driven by niche processes rather than by neutrality. At least for some sets of communities, neutral models appear to explain some classical ecological patterns such as species abundance curves, species-area curves and the decay of similarity with distance (Bell 2001, Hubbell 2001). The patterns claimed to be successfully described by neutral theory have so far been static rather than dynamic. Several studies (McGill et al. 2006, Clark 2009, 2012, Magurran 2011) have repeatedly emphasized the urgent need for studies that analyze patterns and mechanisms of community dynamics instead of studies concerned with snapshots of community structure at a given point in time and space. In addition to this study, several other studies of temporal and spatiotemporal community dynamics show that environmental stochasticity has a major influence on community dynamics (Engen et al. 2002, Lande et al. 2003, Mutshinda et al. 2011, Grøtan et al. 2012). Neutral models do not account for species specific environmental stochasticity and this may in some instances be a shortcoming. For example, consequences of climate change and habitat fragmentation may be different under neutral versus non-neutral models. Thus, an improved understanding of past and future community dynamics requires that effects of environmental variation in space and time are estimated rather than neglected.

Acknowledgements - The study was supported by the Norwegian Research Council (WFR 185109).

\section{References}

Achenbach, L. and Lampert, W. 1997. Effects of elevated temperatures on threshold food concentrations and possible competitive abilities of differently sized cladoceran species. - Oikos 79: 469-476.

Allen, J. et al. 1993. Chaos reduces species extinction by amplifying local population noise. - Nature 364: 229-232.

Astorga, A. et al. 2012. Distance decay of similarity in freshwater communities: do macro- and microorganisms follow the same rules? - Global Ecol. Biogeogr. 88: 365-375.

Bell, G. 2001. Neutral macroecology. - Science 293: 2413-2418.

Bellier, E. et al. 2012. Combining counts and incidence data: an efficient approach to estimate species abundance distribution and diversity indices. - Oecologia 170: 477-488.

Beninca, E. et al. 2011. Resonance of plankton communities with temperature fluctuations. - Am. Nat. 178: E85-E95.

Bjørnstad, O. N. et al. 1999. Spatial population dynamics: analyzing patterns and processes of population synchrony. - Trends Ecol. Evol. 14: 427-432.

Bohonak, A. J. and Jenkins, D. G. 2003. Ecological and evolutionary significance of dispersal by freshwater invertebrates. - Ecol. Lett. 6: 783-796.

Bolker, B. and Grenfell, B. 1993. Impact of vaccination on the spatial correlation and persistence of measles dynamics. - Proc. Natl Acad. Sci. USA 93: 12648-12653.

Caswell, H. 1976. Community structure: a neutral model analysis. - Ecol. Monogr. 46: 327-354.

Clark, J. 2009. Beyond neutral science. - Trend Ecol. Evol. 24: $8-15$.

Clark, J. 2012. The coherence problem with the unified neutral theory of biodiversity. - Trend Ecol. Evol. 24: 198-202.

Condit, R. et al. 2002. Beta-diversity in tropical forest trees. - Science 295: 666-669.

Cottenie, K. 2005. Integrating environmental and spatial processes in ecological community dynamics. - Ecol. Lett. 8: $1175-1182$.

Downing, J. and Rigler, F. 1984. A manual on methods for the assesment of secondary productivity in fresh waters. - Blackwell.

Efron, B. and Tibshirani, R. 1993. An introduction to the bootstrap. Monographs on statistics and applied probability 57. - Chapman and Hall.

Engen, S. and Lande, R. 1996. Population dynamic models generating the lognormal species abundance distribution. - Math. Biosci. 173: 85-102.

Engen, S. and Sxther, B.-E. 2005. Generalizations of the Moran effect explaining spatial synchrony in population fluctuations. - Am. Nat. 166: 603-612.

Engen, S. et al. 2002. Analyzing spatial structure of communities using the two-dimensional poisson lognormal species abundance model. - Am. Nat. 160: 60-73.

Engen, S. et al. 2005. Estimating the pattern of synchrony in fluctuating populations. - J. Anim. Ecol. 74: 601-611.

Engen, S. et al. 2011a. Disentangling the effects of heterogeneity, stochastic dynamics and sampling in a 
community of aquatic insects. - Ecol. Model. 222: 1387-1393.

Engen, S. et al. 2011b. Estimating similarity of communities: a parametric approach to spatio-temporal analysis of species diversity. - Ecography 34: 220-231.

Etienne, R. S. 2007. A neutral sampling formula for multiple samples and an 'exact' test of neutrality. - Ecol. Lett. 10: 608-618.

Etienne, R. S. et al. 2007. Modes of speciation and the neutral theory of biodiversity. - Oikos 116: 241-258.

Fischer, J. M. et al. 2001. Compensatory dynamics in zooplankton community responses to acidification: measurement and mechanisms. - Ecol. Appl. 11: 1060-1072.

Gilbert, B. and Lechowicz, M. J. 2004. Neutrality, niches, and dispersal in a temperate forest understory. - Proc. Natl Acad. Sci. USA 101: 7651-7656.

Gillooly, J. F. 2000. Effect of body size and temperature on generation time in zooplankton. - J. Plankton Res. 22: 241-251.

Grenfell, B. T. et al. 1998. Noise and determinism in synchronized sheep dynamics. - Nature 394: 674-677.

Grøtan, V. and Engen, S. 2008. poilog: Poisson lognormal and bivariate Poisson lognormal distribution. - R package ver. $0.4,<$ http://cran.r-project.org/web/packages/ poilog/>.

Grøtan, V. et al. 2005. Climate causes large-scale spatial synchrony in population fluctuations of a temperate herbivore. - Ecology 86: 1472- 1482.

Grøtan, V. et al. 2012. Seasonal cycles of species diversity and similarity in a tropical butterfly community. - J. Anim. Ecol. 81: 714-723.

Halvorsen, G. et al. 2004. Zooplankton in Lake Atnsjoen 1985-1997. - Hydrobiologia 521: 149-175.

Heino, M. et al. 1997. Synchronous dynamics and rates of extinction in spatially structured populations. - Proc. R. Soc. B 264: 481-486.

Hessen, D. O. et al. 2006. Extrinsic and intrinsic controls of zooplankton diversity in lakes. - Ecology 87: 433-443.

Hillebrand, H. et al. 2008. Consequences of dominance: a review of evenness effects on local and regional ecosystem processes. - Ecology 89: 1510-1520.

Houlahan, J. E. et al. 2007. Compensatory dynamics are rare in natural ecological communities. - Proc. Natl Acad. Sci. USA 104: 3273-3277.

Hubbell, S. 2001. The unified neutral theory of biodiversity and biogeography. - Princeton Univ. Press.

Hutchinson, G. 1957. Concluding remarks - Cold Spring Harbor Symposia on Quantitative Biology 22: 415-427. Reprinted in 1991: classics in theoritical biology. - Bull. Math. Biol. 53: $193-213$.

Ives, A. R. and Cardinale, B. J. 2004. Food-web interactions govern the resistance of communities after non-random extinctions. - Nature 429: 174-177.

Jenkins, D. G. and Underwood, M. O. 1998. Zooplankton may not disperse readily in wind, rain, or waterfowl. - Hydrobiologia 388: 15-21.

Keitt, T. H. 2008. Coherent ecological dynamics induced by large-scale disturbance. - Nature 454: 331-334.

Keller, W. 2007. Implications of climate warming in boreal shield lakes: a review and synthesis. - Environ. Rev. 15: 99-112.

Klug, J. L. et al. 2000. Compensatory dynamics in planktonic community responses to ph perturbations. - Ecology 81: 387-398.

Lande, R. et al. 1999. Spatial scale of population synchrony: environmental correlation versus dispersal and density regulation. - Am. Nat. 154: 271-281.
Lande, R. et al. 2003. Stochastic population dynamics in ecology and conservation. Oxford series in ecology and evolution. - Oxford Univ. Press.

Leibold, M. A. et al. 2004. The metacommunity concept: a framework for multi-scale community ecology. - Ecol. Lett. 7: 601-613.

Lindstrom, J. et al. 1996. Large-scale synchrony in the dynamics of capercaillie, black grouse and hazel grouse populations in finland. - Oikos 76: 221-227.

Magurran, A. 2011. Measurement biological diversity in time (and space). - In: Magurran, A. and McGill, B. (eds), Biological diversity: frontiers in measurement and assesment. Oxford Univ. Press, pp. 85-93.

Maloney, K. O. and Munguia, P. 2011. Distance decay of similarity in temperate aquatic communities: effects of environmental transition zones, distance measure, and life histories. - Ecography 34: 287-295.

McGill, B. et al. 2006. Empirical evaluation of neutral theory. - Ecology 87: 1411-1423.

Moran, P. 1953. Global patterns of environmental synchrony and the moran effect. - Aust. J. Zool. 1: 291-298.

Mutshinda, C. M. et al. 2011. A multispecies perspective on ecological impacts of climatic forcing. - J. Anim. Ecol. 80: 101-107.

Nekola, J. C. and White, P. S. 1999. The distance decay of similarity in biogeography and ecology. - J. Biogeogr. 26: 867-878.

Ranta, E. et al. 1995. Synchrony in population dynamics. - Proc. R. Soc. B 262: 113-118.

Ranta, E. et al. 1997a. The Moran effect and synchrony in population dynamics. - Oikos 78: 136-142.

Ranta, E. et al. 1997b. The spatial dimension in population fluctuations. - Science 278: 1621-1623.

Ranta, E. et al. 1999. Spatially autocorrelated disturbances and patterns in population synchrony. - Proc. R. Soc. B 288: $1851-1856$.

Ranta, E. et al. 2008. Detecting compensatory dynamics in competitive communities under environmental forcing. - Oikos 117: 1907-1911.

Rosindell, J. et al. 2011. The unified neutral theory of biodiversity and biogeography at age ten. - Trends Ecol. Evol. 26: 340-348.

Sæther, B.-E. et al. 2007. The extended moran effect and large-scale synchronous fluctuations in the size of great tit and blue tit populations. - J. Anim. Ecol. 76: 315-325.

Sæther, B.-E. et al. 2013. Species diversity and community similarity in fluctuating environments: parametric approaches using species abundance distributions. - J. Anim. Ecol. 82:721-728.

Shurin, J. B. 2000. Dispersal limitation, invasion resistance, and the structure of pond zooplankton communities. - Ecology 81: 3074-3086.

Shurin, J. B. et al. 2010. Environmental stability and lake zooplankton diversity - contrasting effects of chemical and thermal variability. - Ecol. Lett. 13: 453-463.

Skaugen, T. et al. 2003. Adjustment of dynamically downscaled temperature and precipitation data in norway. - Tech. Rep., Norwegian Meteorological Inst.

Soininen, J. et al. 2007. The distance decay of similarity in ecological communities. - Ecography 30: 3-12.

Steiner, C. F. et al. 2011. Dispersal promotes compensatory dynamics and stability in forced metacommunities. - Am. Nat. 178: 159- 170.

Thompson, P. and Shurin, J. 2011. Regional zooplankton biodiversity provides limited buffering of pond ecosystems against climate change. - J. Anim. Ecol. 81: 251-259. 
Travis, J. J. 2003. Climate change and habitat destruction: a deadly anthropogenic cocktail. - Proc. R. Soc. B 270: 467-473.

Vasseur, D. A. and Gaedke, U. 2007. Spectral analysis unmasks synchronous and compensatory dynamics in plankton communities. - Ecology 88: 2058-2071.

Vasseur, D. A. et al. 2005. A seasonal alternation of coherent and compensatory dynamics occurs in phytoplankton. - Oikos 110: $507-514$.

Supplementary material (Appendix ECOG-00175 at $<$ www.oikosoffice.lu.se/appendix $>$ ). Appendix A-B.
Vyverman, W. et al. 2007. Historical processes constrain patterns in global diatom diversity. - Ecology 88: 1924-1931.

Walseng, B. et al. 2006. Major contribution from littoral crustaceans to zooplankton species richness in lakes. - Limnol. Oceanogr. 51: 2600-2606.

Yan, N. D. et al. 2007. Changes in zooplankton and the phenology of the spiny water flea, bythotrephes, following its invasion of Harp Lake, Ontario, Canada. - Can. J. Fish. Aquat. Sci. 58: 2341- 2350. 